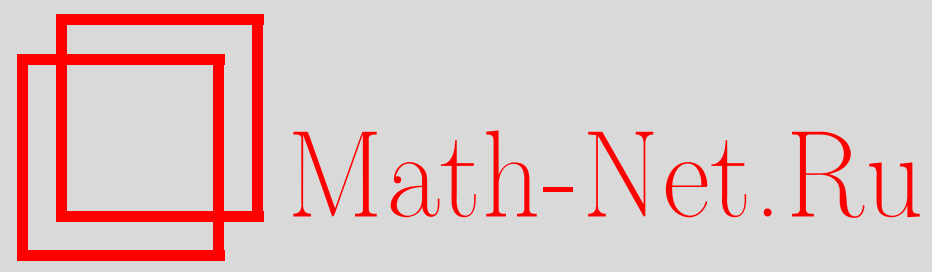

В. Н. Салий, Квазибулевы степени элементарных 2-групп, Матем. заметки, 2001, том 69, выпуск 6, 899-905

DOI: https://doi.org/10.4213/mzm703

Использование Общероссийского математического портала Math-Net.Ru подразумевает, что вы прочитали и согласны с пользовательским соглашением http://www.mathnet.ru/rus/agreement

Параметры загрузки:

IP: 52.23 .180 .231

26 апреля 2023 г., 04:15:40

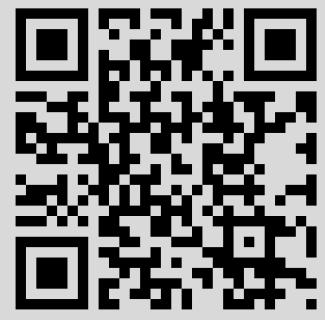


УДК 512.532

\title{
КВАЗИБУЛЕВЫ СТЕПЕНИ ЭЛЕМЕНТАРНЫХ 2-ГРУПП
}

\section{В.Н. Салий}

\begin{abstract}
Охарактеризованы группоиды с единицей, допускающие вложение в квазибулевы степени элементарных 2-групп.

Библиографияя: 5 названий.
\end{abstract}

В [1] были описаны групподы с единицей, вложимые в квазибулевы степени элементарных абелевых $p$-групп при $p>2$. Случай $p=2$ вьпадает из общей схемы и оставался не рассмотренным. В настоящей заметке этот пробел заполняется: получена характеризация группоидов с единицей, допускающих вложение в квазибулевы степени элементарных 2-групп. Тем самьм, полностью завершено исследование минимальных многообразий полугрупп в обсуждаемом аспекте (подробности см. в [1]).

Напомним основные понятия.

Ортогональной системой в полной решетке $L$ назьвается подмножество $\left\{l_{i} \mid i \in I\right\}$ такое, что $l_{i} \wedge l_{j}=0$ для $i \neq j$ и $\bigvee_{i \in I} l_{i}=1$ (здесь 0 и $1-$ наименьший и наибольший элементы в $L$ соответственно). Ортогональная система $\left\{l_{i} \mid i \in I\right\}$ по определению независима, если $\bigvee_{j \in J} l_{j} \wedge \bigvee_{k \in K} l_{k}=0$ для любого разбиения $I=J \cup K, J \cap K=\varnothing$. Полная решетка с дополнениями называется квазибулевой, если в ней все ортогональные системы независимы. Класс квазибулевых решеток содержит, в частности, все полные булевы решетки. Полная решетка с дополнениями тогда и только тогда будет квазибулевой, когда она допускает ^-гомоморфизм на полную булеву решетку, которьй сохраняет точные верхние грани ортогональных систем и, кроме того, взаимно однозначен в 0 и 1 (см. [2] и [3, с. 271]). Такой ^-гомоморфизм называется каноническим.

Пусть $(G, \cdot)$ - группа, т.е. полугруппа с единицей, где каждый элемент имеет обратный, и $L$ - квазибулева решетка. Под $L$-степенью группы $G$ понимается совокупность $G[L]$ всех отображений $\nu: G \rightarrow L$ таких, что $\{\nu(g) \mid g \in G\}$ является ортогональной системой в $L$, снабженной умножением

$$
(\mu \nu)(f)=\bigvee_{f=g h}(\mu(g) \wedge \nu(h))
$$

(для любых $\mu, \nu \in G[L], f \in G$ ). Когда $L$ пробегает класс всех квазибулевых решеток, получаем квазибулевы степени групшы $G$, среди которых находятся и все ее булевы степени.

Булева степень любой группы является группой. Как показано в [1], все квазибулевы степени группы оказываются ассоциациями. Ассоииация - это группоид с единицей, в котором каждый элемент имеет обратный и умножение ассоциативно в единице. Группоид $A$ с единицей $е$ тогда и только тогда будет ассоциацией, когда он допускает взаимно 
однозначньй в единице гомоморфизм на группу. Ядром такого гомоморфизма является конгруэнция $\theta$, состоящая из пар совместно обратимых элементов, т.е. элементов, имеющих одни и те же обратные [2].

Группа $G$ назьвается әлементарной 2-группой, если порядок любого ее неединичного элемента равен 2. Элементарная 2-группа коммутативна.

Следующая теорема описьвает группоиды с единицей, вложимые в квазибулевы степени элементарных 2-групा.

Теорема. Группоид $A$ с единичей е тогда и только тогда вкладывается в подходящую квазибулеву степень некоторой әлементарной 2-группь, когда

I) әруппоид $A$ является коммутативной ассочиачией, в которой выполняется тохсдество $\alpha \alpha=e$;

II) на А существует стабильный порядок $\leqslant$, тождественно удовлетворяющий неравенствам

1) $\alpha \beta \leqslant \alpha(\alpha(\alpha \beta))$

2) $\alpha \beta \leqslant(\alpha(\alpha \beta))(\beta(\alpha \beta))$

3) $\alpha \beta \leqslant((\alpha \beta) \beta)((\alpha \beta)((\alpha \beta) \beta))$;

4) $\alpha \beta \leqslant((\alpha \beta)((\alpha \beta) \alpha))((\alpha \beta)((\alpha \beta) \beta))$.

ДокАЗАТЕЛЬСтво. Необходимость. Пусть $A$ - подгруппоид с единицей (подассоциация) ассоциации $G[L]$, где $G-2$-группа, $L$ - квазибулева решетка. Так как $G$ - коммутативная группа, умножение в $G[L]$, определенное формулой $(1)$, также коммутативно. Квадрат каждого элемента из $A$ равен единице:

$$
(\mu \mu)(e)=\bigvee_{e=f g}(\mu(f) \wedge \mu(g))=\bigvee_{f} \mu(f)=1
$$

и $(\mu \mu)(f)=0$ для любого $f \neq e$. Таким образом, условие I) вьполняется во всех квазибулевых степенях элементарных 2-групп. Возможны два случая.

1. Пусть $A / \theta \cong Z_{2}$. В этом случае в $A$ произведение любых двух нединичных элементов равняется единице $e$. Порядком на $A$, удовлетворяющим условиям II), является, например, тождественное отношение $\Delta$.

2. Пусть $A / \theta \nsucceq Z_{2}$. Заметим, что любые два неединичных элемента $f, g$ в элементарной 2-группе, отличной от $Z_{2}$, порождают четверную группу $\{e, f, g, f g\}$.

Покажем, что в $G[L]$, где $G \not Z_{2}$, условиям II) удовлетворяет естественный порядок $\mu \leqslant \nu \Longleftrightarrow(\forall f \in G)(\mu(f) \leqslant \nu(f))$. Очевидно, что этот порядок стабилен относительно умножения в $G[L]$, определенного формулой $(1)$.

ЛЕмма 1. Для любых $\mu, \nu \in G[L] u f, g \in G$ выполняется неравенство

$$
\mu(f) \wedge(\mu \nu)(g) \geqslant \mu(f) \wedge \nu(f g) .
$$

ДокАЗАТЕЛЬСТво. В самом деле,

$$
\begin{aligned}
\mu(f) \wedge(\mu \nu)(g) & =\mu(f) \wedge \bigvee_{g=u v}(\mu(u) \wedge \nu(v)) \\
& =\mu(f) \wedge\left((\mu(f) \wedge \nu(f g)) \vee \bigvee_{g=u v, u \neq f}(\mu(u) \wedge \nu(v))\right. \\
& \geqslant \mu(f) \wedge(\mu(f) \wedge \nu(f g))=\mu(f) \wedge \nu(f g) .
\end{aligned}
$$


Лемма доказана.

Используя лемму 1 , проверяем выполнимость в $G[L]$ неравенств 1$)-4)$ из условий II).

1) Рассмотрим

$$
\begin{aligned}
(\mu(\mu(\mu \nu)))(f) & =\bigvee_{f=g h}(\mu(g) \wedge(\mu(\mu \nu))(h)) \geqslant \bigvee_{f=g h}(\mu(g) \wedge(\mu \nu)(g h)) \\
& \geqslant \bigvee_{f=g h}(\mu(g) \wedge \nu(g g h))=\bigvee_{f=g h}(\mu(g) \wedge \nu(h))=(\mu \nu)(f),
\end{aligned}
$$

т.e. $\mu \nu \leqslant \mu(\mu(\mu \nu))$.

2) Рассмотрим

$$
\begin{aligned}
((\mu(\mu \nu))(\nu(\mu \nu)))(f) & =\bigvee_{f=g h}((\mu(\mu \nu))(g) \wedge(\nu(\mu \nu))(h)) \\
& =\bigvee_{f=g h}\left(\left(\bigvee_{g=u v}(\mu(u) \wedge(\mu \nu)(v))\right) \wedge\left(\bigvee_{h=x y}(\nu(x) \wedge(\mu \nu)(y))\right)\right) \\
& \geqslant \bigvee_{f=g h}\left(\left(\bigvee_{g=u v}(\mu(u) \wedge \nu(u v))\right) \wedge\left(\bigvee_{h=x y}(\nu(x) \wedge \mu(x y))\right)\right) \\
& =\bigvee_{f=g h}\left(\left(\bigvee_{u}(\mu(u) \wedge \nu(g))\right) \wedge\left(\bigvee_{x}(\nu(x) \wedge \mu(h))\right)\right) \\
& \geqslant \bigvee_{f=g h}(\mu(h) \wedge \nu(g))=(\nu \mu)(f)=(\mu \nu)(f),
\end{aligned}
$$

т.е. $\mu \nu \leqslant(\mu(\mu \nu))(\nu(\mu \nu))$.

3) Рассмотрим

$$
\begin{aligned}
& (((\mu \nu) \nu)((\mu \nu)((\mu \nu) \nu)))(f)=\bigvee_{f=g h}(((\mu \nu) \nu)(g) \wedge((\mu \nu)((\mu \nu) \nu))(h)) \\
& =\bigvee_{f=g h}\left(\left(\bigvee_{g=u v}((\mu \nu)(u) \wedge \nu(v))\right) \wedge \bigvee_{h=x y}((\mu \nu)(x) \wedge((\mu \nu) \nu)(y))\right) \\
& \geqslant \bigvee_{f=g h}\left(\left(\bigvee_{g=u v}(\mu(u v) \wedge \nu(v))\right) \wedge \bigvee_{h=x y}((\mu \nu)(x) \wedge \nu(x y))\right) \\
& =\bigvee_{f=g h}\left(\left(\bigvee_{v}(\mu(g) \wedge \nu(v))\right) \wedge \bigvee_{x}((\mu)(x) \wedge \nu(h))\right) \\
& \geqslant \bigvee_{f=g h}\left((\mu(g) \wedge \nu(h)) \wedge \bigvee_{x}(\mu(x h) \nu(h))\right) \\
& \geqslant \bigvee_{f=g h}(\mu(g) \wedge \nu(h))=(\mu \nu)(f)
\end{aligned}
$$

т.е. $\mu \nu \leqslant((\mu \nu) \nu)((\mu \nu)((\mu \nu) \nu))$.

4) Рассмотрим

$$
((\mu \nu)((\mu \nu) \mu))((\mu \nu)((\mu \nu) \nu))(f)=\bigvee_{f=g h}(((\mu \nu)((\mu \nu) \mu))(g) \wedge((\mu \nu)((\mu \nu) \nu))(h))
$$




$$
\begin{aligned}
= & \bigvee_{f=g h}\left(\left(\bigvee_{g=u v}((\mu \nu)(u) \wedge((\mu \nu) \mu)(v))\right) \wedge \bigvee_{h=x y}((\mu \nu)(x) \wedge((\mu \nu) \nu)(y))\right) \\
\geqslant & \bigvee_{f=g h}\left(\left(\bigvee_{g=u v}((\mu \nu)(u) \wedge \mu(u v))\right) \wedge\left(\bigvee_{h=x y}((\mu \nu)(x) \wedge \nu(x y))\right)\right) \\
= & \bigvee_{f=g h}\left(\left(\bigvee_{u}((\mu \nu)(u) \wedge \mu(g))\right) \wedge\left(\bigvee_{x}((\mu \nu)(x) \wedge \nu(h))\right)\right) \\
\geqslant & \bigvee_{f=g h}\left(\left(\bigvee_{u}(\mu(g) \wedge \nu(g u))\right) \wedge\left(\bigvee_{x}(\mu(x h) \wedge \nu(h))\right)\right) \\
\geqslant & \bigvee_{f=g h}(\mu(g) \wedge \nu(h))=(\mu \nu)(f),
\end{aligned}
$$

т.е. $\mu \nu \leqslant((\mu \nu)((\mu \nu) \mu))((\mu \nu)((\mu \nu)((\mu \nu) \nu)))$.

Таким образом, квазибулевы степени элементарных 2-групп обладают и всеми свойствами II).

Достаточность. Пусть группоид $A$ с единицей $e$ удовлетворяет условиям I), II). Так как выполняется I), отношение $\theta$ совместной обратимости является конгруэнцией на $A$ и факторассоциация $G=A / \theta$ является элементарной 2 -группой.

1. Рассмотрим случай $G=Z_{2}=\{e, z\}$.

Тогда произведение любых двух неединичных элементов в $A$ равно $e$ и стабильньм порядком, удовлетворяющим аксиомам II), будет, например, тождественное отношение $\Delta$.

Пусть $A=\{e\} \cup\left\{\alpha_{i} \mid i \in I\right\}$. Через $M_{|I|}$ обозначим решетку высоты 2 с наименьшим элементом $\alpha^{0}$, наибольшим элементом $\alpha^{1}$ и атомами $\alpha_{i}, i \in I$. В четырехэлементной булевой алгебре $L^{*}=\{0, a, b, 1\}$ заменим атом $b$ решеткой $M_{|I|}$. При этом считается, что элемент $\alpha^{0}$ непосредственно следует за нулем 0 , а единица 1 непосредственно следует за $\alpha^{1}$. Получается квазибулева решетка $L$.

Каждому элементу $\alpha$ из $A$ сопоставим отображение $\nu_{\alpha}: Z_{2} \rightarrow L$ следующим образом: $\nu_{e}=(0,1), \nu_{\alpha_{i}}=\left(a, \alpha_{i}\right), i \in I$ (функция $\nu: Z_{2} \rightarrow L$ записывается в виде вектора $(\nu(e), \nu(z)))$. Очевидно, что соответствие $\alpha \mapsto \nu_{\alpha}$ взаимно однозначно отображает $A$ в $Z_{2}[L]$. При этом $\nu_{\alpha} \nu_{e}=\nu_{\alpha}$ для любого $\alpha \in A$ и вьполнены соотношения

$$
\begin{aligned}
\left(\nu_{\alpha_{i}} \nu_{\alpha_{j}}\right)(e) & =\bigvee_{e=x y}\left(\nu_{\alpha_{i}}(x) \wedge \nu_{\alpha_{j}}(y)\right)=\left(\nu_{\alpha_{i}}(e) \wedge \nu_{\alpha_{j}}(e)\right) \vee\left(\nu_{\alpha_{i}}(z) \wedge \nu_{\alpha_{j}}(z)\right) \\
& =(a \wedge a) \vee\left(\alpha_{i}^{0} \wedge \alpha_{j}^{0}\right)=a \vee \alpha^{0}=1 \\
\left(\nu_{\alpha_{i}} \nu_{\alpha_{j}}\right)(z) & =\left(\nu_{\alpha_{i}}(e) \wedge \nu_{\alpha_{j}}(z)\right) \vee\left(\nu_{\alpha_{i}}(z) \wedge \nu_{\alpha_{j}}(e)\right)=\left(a \wedge \alpha_{j}\right) \vee\left(\alpha_{i} \wedge a\right)=0
\end{aligned}
$$

т.e. $\nu_{\alpha_{i}} \nu_{\alpha_{j}}=\nu_{e}=\nu_{\alpha_{i} \alpha_{j}}$.

Таким образом, любая ассоциация $A$ с $A / \theta \cong Z_{2}$ вкладьвается в квазибулеву степень группы $Z_{2}$.

2. Пусть $G=A / \theta \nsucceq Z_{2}$. Элементарная 2-группа $G$ является прямьм произведением циклических групп $G_{k}, k \in K$, каждая из которых изоморфна $Z_{2}$ [4, теорема 8.5]. Элементы группы $G$ можно считать функциями $f: K \rightarrow Z_{2}$, для которых множества $\operatorname{supp} f:=\{k \in K \mid f(k) \neq e\}$ конечны. Группа $G$ вкладьвается в группу характеров $\operatorname{Hom}\left(G, Z_{2}\right)$. Если $\chi_{f}: G \rightarrow Z_{2}$ - характер, соответствующий элементу $f \in G$, то $\nu_{f}=\chi_{f}^{-1}$ отображает $Z_{2}$ в булеву алгебру $L^{*}=P(G)$ всех подмножеств множества $G$. 
Понятно, что $\chi_{f}^{-1}(e) \cap \chi_{f}^{-1}(z)=\varnothing$ и $\chi_{f}^{-1}(e) \cup \chi_{f}^{-1}(z)=G$, так что $\nu_{f} \in Z_{2}\left[L^{*}\right]$. Несложные выкладки показьвают, что $\left(\nu_{f} \nu_{g}\right)(e)=\left(\nu_{f}(e) \cap \nu_{g}(e)\right) \cup\left(\nu_{f}(z) \cap \nu_{g}(z)\right)=\nu_{f g}(e)$ и $\left(\nu_{f} \nu_{g}\right)(z)=\left(\nu_{f}(e) \cap \nu_{g}(z)\right) \cup\left(\nu_{f}(z) \cap \nu_{g}(e)\right)=\nu_{f g}(z)$ и, значит, соответствие $f \mapsto \nu_{f}$ является вложением групшы $G=A / \theta$ в булеву степень $Z_{2}\left[L^{*}\right]$, где $L^{*}=P(G)$.

Трансформацией этого представления и получается вложение группоида $A$ в группоид $Z_{2}[L]$, где $L-$ квазибулева решетка, получающаяся из $L^{*}$ с помощью описьваемых ниже конструкций.

Под идеалами упорядоченного множества понимаются его минорантно насьщенные подмножества.

Пусть $\alpha \in A$. Через $L(\alpha)$ обозначим решетку $\operatorname{Id}(\theta(\alpha))$ всех идеалов (включая пустой) упорядоченного множества $(\theta(\alpha), \leqslant)$, где $\leqslant-$ стабильный порядок на $A$, существование которого постулируется в II). В булевой алгебре $L^{*}=P(G)$ каждьй элемент вида $\chi_{\theta(\alpha)}^{-1}(x), x \in Z_{2}$, заменим решеткой $L(\alpha)$, обозначая ее в этой ситуации через $L(\alpha, x)$. Каждьй элемент $l$, лежащий в $L^{*}$ ниже элемента вида $\chi_{\theta(\alpha)}^{-1}(x), x \in Z_{2}$, и отличньй от $\varnothing$, заменим решеткой $P(\bar{\theta})$ всех подмножеств отношения $\bar{\theta}=(A \times A)-\theta$, обозначая ее в этой ситуации через $L(l)$.

Теперь определим порядок в множестве $L$, полученном из $L^{*}$ в результате всех указанных замен. Сохраняя все порядковые связи, имеющиеся в решетках вида $L(\alpha, x)$ и $L(l)$, зададим следующие соотношения:

а) наименьший элемент 0 решетки $L^{*}$ (т.е. $\varnothing$ ) остается наименьшим элементом в $L$;

б) если $\chi_{\theta(\alpha)}^{-1}(x)<l$ в $L^{*}$, то в $L$ каждый элемент подрешетки $L(\alpha, x)$ считается меньше $l$;

в) если $0<l_{1}<l_{2}<\chi_{\theta(\alpha)}^{-1}(x)$ в $L^{*}$ для некоторых $\alpha$ и $x$, то каждый элемент подрешетки $L\left(l_{1}\right)$ считается меньшим одноименного элемента подрешетки $L\left(l_{2}\right)$;

г) если $0<l<\chi_{\theta(\alpha)}^{-1}(x)$ в $L^{*}$ для некоторьх $\alpha$ и $x$, то наибольшим минорантом элемента $J \in L(\alpha, x)$ в подрешетке $L(l)$ считается отношение

$$
\rho(J)=\bigcup_{\beta \in J}\{(\gamma, \delta) \in \bar{\theta} \mid \beta \in\langle\gamma, \delta\rangle \uparrow\}
$$

где $\langle\gamma, \delta\rangle$ - подгруппоид в $A$, порожденный элементами $\gamma, \delta$;

д) если $0<l<\chi_{\theta(\alpha)}^{-1}(x)$ в $L^{*}$ для некоторых $\alpha$ и $x$, то наименьшим мажорантом элемента $\rho \in P(\bar{\theta})$ в подрешетке $L(\alpha, x)$ считается идеал $J(\rho)=\bigcup_{(\gamma, \delta) \in \rho} J(\gamma, \delta)$, где $J(\gamma, \delta)=\downarrow$ least $(\langle\gamma, \delta\rangle \cap \theta(\alpha))$, если этот наименьший элемент существует (стрелки $\downarrow$ и $\uparrow$ обозначают соответственно минорантное и мажорантное насыщение);

е) для $l_{1}, l_{2}$, лежаших в $L^{*}$ выше элемента вида $\chi_{\theta(\alpha)}^{-1}(x)$, в $L$ сохраняется упорядоченность, связьвающая их в $L^{*}$.

Транзитивное замыкание построенного отношения, состоящего из объединения порядков в решетках вида $L(\alpha, x)$ и $L(l)$ и неравенств, заданных в а)-e), упорядочивает множество $L$.

Покажем, что множество $L$ с введенным порядком - решетка. Здесь нужно рассмотреть два случая.

1. Пусть $J_{1}, J_{2} \in L(\alpha, x)$. В $L(\alpha, x)$ наибольшей нижней гранью для $\left\{J_{1}, J_{2}\right\}$ будет $J_{1} \cap J_{2}$. Но у этих идеалов есть обшие нижние грани и в каждой подрешетке вида $L(l)$, где $l<\chi_{\theta(\alpha)}^{-1}(x)$ в $L^{*}$. Пусть $\chi_{\theta(\alpha)}^{-1}(x)$ является покрытием для $l$ в $L^{*}$. Наибольшей 
нижней гранью для $\left\{J_{1}, J_{2}\right\}$ в $L(l)$ будет пересечение отношений

$$
\rho\left(J_{i}\right)=\bigcup_{\beta \in J_{i}}\{(\gamma, \delta) \in \bar{\theta} \mid \beta \in\langle\gamma, \delta\rangle \uparrow\}, \quad i=1,2 .
$$

Пусть $(\gamma, \delta) \in \rho\left(J_{1}\right) \cap \rho\left(J_{2}\right)$. Если в $\langle\gamma, \delta\rangle \cap \theta(\alpha)$ нет наименьшего элемента, то $J(\gamma, \delta)=\varnothing$ и, значит, $J(\gamma, \delta) \subseteq J_{1} \cap J_{2}$. Пусть $\alpha_{0}=\operatorname{least}(\langle\gamma, \delta\rangle \cap \theta(\alpha))$. Так как $(\gamma, \delta) \in \rho\left(J_{1}\right)$, то существует элемент $\alpha_{1} \in J_{1}$ такой, что $\alpha_{1} \in\langle\gamma, \delta\rangle \uparrow \cap \theta(\alpha)$. Аналогично в $J_{2}$ имеется элемент $\alpha_{2} \in\langle\gamma, \delta\rangle \uparrow \cap \theta(\alpha)$. Но тогда $\alpha_{0} \leqslant \alpha_{1}$ и $\alpha_{0} \leqslant \alpha_{2}$ и, следовательно, $J(\gamma, \delta)=\downarrow$ least $(\langle\gamma, \delta\rangle \cap \theta(\alpha)) \subseteq J_{1} \cap J_{2}$. Таким образом, $J(\gamma, \delta) \subseteq J_{1} \cap J_{2}$ для любой пары $(\gamma, \delta)$ из $\rho\left(J_{1}\right) \cap \rho\left(J_{2}\right)$. Отсюда $\rho\left(J_{1}\right) \cap \rho\left(J_{2}\right)<J_{1} \cap J_{2}$ в $L$, так чтоinf $L\left\{J_{1}, J_{2}\right\}=J_{1} \cap J_{2}$.

2 . Пусть $\rho_{1}, \rho_{2} \in L(l)$. В $L(l)$ наименьшей верхней гранью для $\left\{\rho_{1}, \rho_{2}\right\}$ будет $\rho_{1} \cup \rho_{2}$. Но у этих множеств (т.е. отношений, содержащихся в $\bar{\theta}$ ) есть общие верхние грани и в каждой подрешетке вида $L(\alpha, x)$, где $l<\chi_{\theta(\alpha)}^{-1}(x)$ в $L^{*}$. Пусть $\chi_{\theta(\alpha)}^{-1}(x)$ является покрытием для $l$ в $L^{*}$. Наименьшей верхней гранью для $\left\{\rho_{1}, \rho_{2}\right\}$ в $L(\alpha, x)$ будет объединение идеалов $J\left(\rho_{i}\right)=\bigcup_{(\gamma, \delta) \in \rho_{i}} J(\gamma, \delta), i=1,2$. Пусть $(\gamma, \delta) \in \rho_{1} \cup \rho_{2}$. Если $(\gamma, \delta) \in \rho_{i}$, то, очевидно, $J(\gamma, \delta) \subseteq J\left(\rho_{i}\right), i \in\{1,2\}$, откуда $\rho_{1} \cup \rho_{2}<J\left(\rho_{1}\right) \cup J\left(\rho_{2}\right)$, так что $\sup _{L}\left\{\rho_{1}, \rho_{2}\right\}=\rho_{1} \cup \rho_{2}$.

Мы получили, что $L$ - решетка. Очевидно, что она будет полной. Если в $L$ отождествить элементы каждой подрешетки вида $L(\alpha, x)$ и отождествить элементы каждой подрешетки вида $L(l)$, получится канонический $\wedge$-гомоморфизм решетки $L$ на булеву решетку $L^{*}=P(G)$. Следовательно, $L-$ квазибулева решетка.

Для дальнейшего потребуется

ЛЕмма 2. Имеет место соотношение $\gamma \delta=\operatorname{least}(\langle\gamma, \delta\rangle \cap \theta(\gamma \delta))$.

ДокАЗАТЕЛЬСТво. Нужно доказать, что $\gamma \delta$ является наименьшим среди элементов подгруппоида $\langle\gamma, \delta\rangle$, входящих в $\theta$-класс, содержаший $\gamma \delta$.

Доказательство ведется индукцией по длине слов в алфавите $\{\gamma, \delta\}$, выражающих элементы 2-порожденного подгруппида $\langle\gamma, \delta\rangle$. Запись $[\alpha]$ означает “слово из $\theta(\alpha)$ ". Заметив, что в элементарной 2-групе любые два неединичных элемента порождают четверную группу, и предположив, что $\gamma \delta \leqslant[\gamma \delta]$ для всех слов из $\langle\gamma, \delta\rangle$ длины меньшей, чем длина данного слова, получаем

$$
\begin{aligned}
{[\gamma \delta] } & =[\gamma][\delta]=([\gamma \delta][\delta])([\gamma \delta][\gamma]) \geqslant((\gamma \delta)[\delta])((\gamma \delta)[\gamma]) \\
& =((\gamma \delta)([\gamma \delta][\gamma]))((\gamma \delta)([\gamma \delta][\delta])) \geqslant((\gamma \delta)((\gamma \delta)[\gamma]))((\gamma \delta)((\gamma \delta)[\delta])) \\
& =((\gamma \delta)((\gamma \delta)([\gamma \delta][\delta])))((\gamma \delta)((\gamma \delta)([\gamma \delta][\gamma]))) \\
& \geqslant((\gamma \delta)((\gamma \delta)((\gamma \delta)[\delta])))((\gamma \delta)((\gamma \delta)((\gamma \delta)[\gamma])))
\end{aligned}
$$

(в предыдущих неравенствах использовались предположение индукции и стабильность порядка $\leqslant$, теперь ссылаемся на неравенство 1) из аксиомы II), а затем снова пойдет цепочка неравенств, продолжающих предыдущую и вытекающих из предположения индукции и стабильности порядка $\leqslant$, и т.д.)

$$
\geqslant((\gamma \delta)[\delta])((\gamma \delta)[\gamma]) \geqslant \cdots
$$

Возможными вариантами окончания этой цепочки могут быть только следующие: $(\gamma(\gamma \delta))((\gamma \delta) \delta),((\gamma \delta) \delta)(\gamma \delta((\gamma \delta) \delta)),((\gamma \delta)(\gamma(\gamma \delta)))((\gamma \delta)((\gamma \delta) \delta))$

Согласно неравенствам 2)-4) из аксиомы II) каждое из этих слов больше, чем $\gamma \delta$. Соответствуюшие неравенства, кроме того, обеспечивают и базис индукции. Лемма доказана. 
Покажем теперь, что группоид $A$ вкладьвается в квазибулеву степень $Z_{2}[L]$. Для большей наглядности элементы подрешеток вида $L(\alpha, x)$ будем записывать в виде пар $\left(J, \chi_{\theta(\alpha)}^{-1}(x)\right)$.

Определим отображение $\varphi: A \rightarrow Z_{2}[L], \alpha \mapsto \nu_{\alpha}$, полагая $\nu_{\alpha}(x)=\left(\alpha \downarrow, \chi_{\theta(\alpha)}^{-1}(x)\right)$, $x \in Z_{2}$, при $\alpha \neq$ и $\nu_{e}=(1,0)$ (здесь $\left.1=G, 0=\varnothing\right)$. Поскольку $\chi_{\theta(\alpha)}^{-1}(e) \cap \chi_{\theta(\alpha)}^{-1}(z)=\varnothing$ и $\chi_{\theta(\alpha)}^{-1}(e) \cup \chi_{\theta(\alpha)}^{-1}(z)=G$, то $\nu_{\alpha}(e) \wedge \nu_{\alpha}(z)=0$ и $\nu_{\alpha}(e) \vee \nu_{\alpha}(z)=1$, откуда $\nu_{\alpha} \in Z_{2}[L]$.

Если $\varphi(\alpha)=\varphi(\beta)$, то $\theta(\alpha)=\theta(\beta)$ и $\alpha \downarrow=\beta \downarrow$, что влечет равенство $\alpha=\beta$. Таким образом, $\varphi$ инъективно.

Нужно доказать, что $\varphi$ - гомоморфизм, т.е. что $\nu_{\alpha} \nu_{\beta}=\nu_{\alpha \beta}$ для любых $\alpha, \beta \in A$.

Случай 1. $(\alpha, \beta) \in \theta$, т.е. $\theta(\alpha)=\theta(\beta)$. Получаем

$$
\begin{aligned}
\left(\nu_{\alpha} \nu_{\beta}\right)(e) & =\left(\nu_{\alpha}(e) \wedge \nu_{\beta}(e)\right) \vee\left(\nu_{\alpha}(z) \wedge \nu_{\beta}(z)\right) \\
& =\left(\alpha \downarrow \cap \beta \downarrow, \chi_{\theta(\alpha)}^{-1}(e)\right) \vee\left(\alpha \downarrow \cap \beta \downarrow, \chi_{\theta(\alpha)}^{-1}(z)\right)=1, \\
\left(\nu_{\alpha} \nu_{\beta}\right)(e) & =\left(\nu_{\alpha}(e) \wedge \nu_{\beta}(z)\right) \vee\left(\nu_{\alpha}(z) \wedge \nu_{\beta}(e)\right)=0,
\end{aligned}
$$

откуда $\nu_{\alpha} \nu_{\beta}=\nu_{e}=\nu_{\alpha \beta}$, так как $\theta(\alpha \beta)=\theta(\alpha) \theta(\beta)=(\theta(\alpha))^{2}=\{e\}$, поскольку $G=A / \theta$ является 2-групшой.

Случай 2. $(\alpha, \beta) \notin \theta$. Имеем

$$
\begin{aligned}
\left(\nu_{\alpha} \nu_{\beta}\right)(e) & =\left(\nu_{\alpha}(e) \wedge \nu_{\beta}(e)\right) \vee\left(\nu_{\alpha}(z) \wedge \nu_{\beta}(z)\right) \\
& =\left(\left(\alpha \downarrow, \chi_{\theta(\alpha)}^{-1}(e)\right) \wedge\left(\beta \downarrow, \chi_{\theta(\beta)}^{-1}(e)\right)\right) \vee\left(\left(\alpha \downarrow, \chi_{\theta(\alpha)}^{-1}(z)\right) \wedge\left(\beta \downarrow, \chi_{\theta(\beta)}^{-1}(z)\right)\right) .
\end{aligned}
$$

При описанном выше вложении группы $G$ в булеву степень $Z_{2}[L]$ вьполняется равен$\operatorname{cтво}\left(\chi_{\theta(\alpha)}^{-1}(e) \cap \chi_{\theta(\beta)}^{-1}(e)\right) \cup\left(\chi_{\theta(\alpha)}^{-1}(z) \cap \chi_{\theta(\alpha)}^{-1}(z)\right)=\chi_{\theta(\alpha \beta)}^{-1}(e)$. Следовательно, $\left(\nu_{\alpha} \nu_{\beta}\right)(e)$ является в $L$ элементом подрешетки $L(\alpha \beta, e)$. Далее, так как $\alpha \in\langle\alpha, \beta\rangle \uparrow$ и $\beta \in\langle\alpha, \beta\rangle \uparrow$, то пара $(\alpha, \beta)$ является обшим минорантом для $\left(\alpha \downarrow, \chi_{\theta(\alpha)}^{-1}(e)\right)$ и $\left(\beta \downarrow, \chi_{\theta(\beta)}^{-1}(e)\right)$ в подрешетке $L\left(\chi_{\theta(\alpha)}^{-1}(e) \cap \chi_{\theta(\beta)}^{-1}(e)\right)$. Из леммы 2 следует, что наименьшим из элементов подрешетки $L(\alpha \beta, e)$, мажорирующих элемент $\{(\alpha, \beta)\}$ из $L\left(\chi_{\theta(\alpha)}^{-1}(e) \cap \chi_{\theta(\beta)}^{-1}(e)\right)$, является главный главный идеал $(\alpha \beta) \downarrow$. Отсюда $\left(\nu_{\alpha} \nu_{\beta}\right)(e)=\left((\alpha \beta) \downarrow, \chi_{\theta(\alpha \beta)}^{-1}(e)\right)=\nu_{\alpha \beta}(e)$.

Аналогично $\left(\nu_{\alpha} \nu_{\beta}\right)(z)=\nu_{\alpha \beta}(z)$.

Этим завершается доказательство теоремы.

Очевидно, что пересечение класса группоидов, описанного в теореме, с многообразием всех полугруп совпадает с многообразием элементарных 2-групп.

Заметим еще, что упомянутое в [1] описание групоидов, вложимых в квазибулевы степени полурешеток, опубликовано в [5].

\section{СПИСОК ЦИТИРОВАННОЙ ЛИТЕРАТУРЫ}

[1] Салий В.Н. Квазибулевы степени элементарных абелевых $p$-групп // Матем. заметки. 1999. T. 66. № 2. C. 264-274.

[2] Salii V. N. Quasi-boolean lattices and associations // Colloq. Math. Soc. János Bolyai. Lectures in Univ. Algebra. Szeged (Hungary), 1983. V. 43. Amsterdam: North-Holland, 1986. P. $429-454$.

[3] Скорняков Л. А. Общая алгебра. Т. 2. М.: Наука, 1991.

[4] Фукс Л. Бесконечные абелевы группы. Т. 1. М.: Мир, 1974.

[5] Салий В. Н. Квазибулевы степени полурешеток // Изв. вузов. Матем. 1999. № 7. С. 54-60.

Саратовский государственный университет им. Н. Г. Черньшевского 